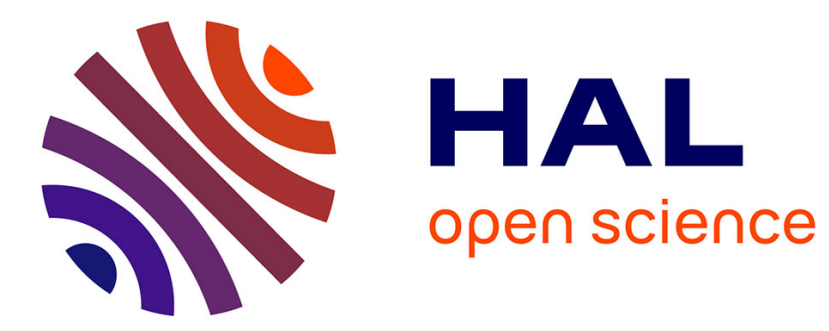

\title{
The influence of rock heterogeneity on the scaling properties of simulated and natural stylolites
}

\author{
M. Ebner, D. Koehn, R. Toussaint, François Renard
}

\section{To cite this version:}

M. Ebner, D. Koehn, R. Toussaint, François Renard. The influence of rock heterogeneity on the scaling properties of simulated and natural stylolites. Journal of Structural Geology, 2009, 31 (1), pp.72 - 82. 10.1016/j.jsg.2008.10.004 . insu-00351889

\section{HAL Id: insu-00351889 \\ https://hal-insu.archives-ouvertes.fr/insu-00351889}

Submitted on 12 Jan 2009

HAL is a multi-disciplinary open access archive for the deposit and dissemination of scientific research documents, whether they are published or not. The documents may come from teaching and research institutions in France or abroad, or from public or private research centers.
L'archive ouverte pluridisciplinaire HAL, est destinée au dépôt et à la diffusion de documents scientifiques de niveau recherche, publiés ou non, émanant des établissements d'enseignement et de recherche français ou étrangers, des laboratoires publics ou privés. 
1

2

3 The influence of rock heterogeneity on the scaling properties of simulated 4 5

6

7 Marcus Ebner*(a), Daniel Koehn (a), Renaud Toussaint (b,c) \& François Renard (d)

\section{and natural stylolites}

\section{5} 6

10

9 8 9

(a) Tectonophysics, Institute of Geosciences, Johannes Gutenberg University, Becherweg 21, D-55099 Mainz, Germany;

(b,c) Institut de Physique du Globe de Strasbourg, UMR CNRS 7516,

3 EOST, Université de Strasbourg I, 5 rue Descartes, F-67084 Strasbourg Cedex, France

4 (d) LGCA-CNRS-Observatoire de Grenoble, Université Joseph Fourier BP 53, F-38041

5 Grenoble, France \& Physics of Geological Processes, University of Oslo, Norway 6 17

*corresponding author: ebnerm@uni-mainz.de

Keywords: stylolites, scaling, roughness, compaction, pressure solution, quenched noise 


\section{Abstract}

Stylolites are among the most prominent deformation patterns in sedimentary rocks that document localized pressure solution. Recent studies revealed that stylolite roughness is characterized by two distinct scaling regimes. The main goal of the present study is to decipher whether this complex scaling behavior of stylolites is caused by the composition of the host rock, i.e. heterogeneities in the material, or is governed by inherent processes on respective scales, namely the transition from a surface energy to an elastic energy dominated regime, as theoretically predicted. For this purpose we have developed a discrete numerical technique, based on a lattice spring model, to simulate the competition between stress, strain, and dissolution during stylolite roughening. We varied systematically the quenched noise, initially present in the material, which controls the roughening. We also changed the size, amount, and dissolution rate of the heterogeneities introduced in our model and evaluated the influence on the scaling exponents. Our findings demonstrate that the roughness and growth exponents are independent of the exact nature of the heterogeneities. We discovered two coinciding crossover phenomena in space and time that separate length and timescales for which the roughening process is either balanced by surface and elastic energies. Our observations are coherent with analytical predictions and with investigations quantifying precisely the scaling laws in the morphology of natural stylolites. The findings presented here can further be used to refine volume loss estimates from the finite strain pattern of stylolites.

\section{Introduction}

Pressure solution in sedimentary rocks results in either intergranular or localized dissolution of material (e.g. Tada \& Siever, 1989). The latter is responsible for the formation of stylolites, a frequent deformation pattern in sedimentary rocks (e. g. Stockdale, 1922; Dunnington 1954, Heald, 1955, Park \& Schot, 1968, Buxton \& Sibley 1981, Rutter, 1983; Railsback, 1993). Stylolites are rough interfaces that frequently contain insoluble material (Fig. 1), which is 
considered to be the residuum of the dissolved rock (Railsback, 1993; and references cited therein). Stylolite initiation is still highly debated (e.g. Tada \& Siever, 1989) but several mechanisms have been proposed that are in agreement with field observations: Formation (I) along preexisting anisotropies (Bathurst, 1987) (II) as anticracks (Fletcher and Pollard, 1981) that propagate due to stress concentrations at anticrack tips (even though this idea was challenged recently by Katsman et al. 2006) and (III) by stress induced self-organization (Merino, 1992, Railsback, 1998; Merino et al., 2006).

In the present study we focus on a quantitative description and characterization of the roughness of simulated stylolites and study their dynamic development independent of the process leading to the initial development of the localization of dissolution along a plane. Based on recent quantitative methods of stylolite roughness characterization (Renard et al., 2004; Schmittbuhl et al., 2004, Koehn et al., 2007, Ebner et al., submitted) we will use statistical tools to compare simulated and natural stylolites. In particular we study the influence of initial heterogeneity concentration in the host-rock on a) stylolite roughness, b) dynamic roughness growth and c) the correlation of crossover phenomena in space and time. To integrate the results of our study in the context of quantitative characterization we will first review some of the major findings and basic principles, used in recent studies, necessary for the understanding of our approach.

The exact classification of stylolites in the field is a difficult task because there is a wide range of geometries (e.g. Park and Schott, 1968) that are often transitional even within a single outcrop. Many previous studies (Park \& Schot, 1968; Buxton \& Sibley, 1981, Guzzetta, 1984; Tada \& Siver, 1989; Railsback, 1993) used classification schemes that were based on visual descriptions of macroscopic features of stylolites. These classification schemes are however not quantitative and hard to compare since these studies focused on a variety of different aspects of stylolite formation. Recent studies, however (Drummond \& Sexton, 1998, Karcz \& Scholz, 2003) took a more quantitative approach using fractal 
concepts to describe the stylolite roughness in a statistical sense. They could describe stylolite roughness with a fractal scaling over several orders of magnitude, which means that their roughness is not dominated by a certain wavelength.

Renard et al. (2004) and Schmittbuhl et al. (2004) went one step further and revealed that bedding parallel stylolite surfaces show a self-affine scaling invariance with characteristic Hurst exponents (also called roughness exponents). A self-affine rough surface is characterized statistically by the fact that points along the surface separated by a distance $\Delta x$ from each other are typically distant in the direction transverse to the surface by $\Delta h=\Delta x^{\alpha}$, where $\alpha$ is the roughness exponent. It was further noticed that two distinct scaling regimes exist that were characterized by two different Hurst or roughness exponents separated by a crossover-length $(L)$, around the millimeter scale for the analyzed natural stylolites. Above this crossover, all investigated stylolites exhibit a Hurst exponent of about 0.5 meaning that they change relatively fast from being flat features on larger scale to being rough features on the smaller scale. Below the crossover-length the Hurst exponent is about 1.0, which means that the slopes, or aspect ratio $\Delta z / \Delta x$, stays more or less constant. Schmittbuhl et al. (2004) and Renard et al. (2004) established from first principles of mechanics and chemistry a model for stylolite growth under the form of a stochastic partial differential equation (called in this case a generalized Langevin equation). This equation simulates the roughening of a stylolite surface as a competition between stabilizing forces (that keep the surface flat), which are controlled by long range elastic and local surface tension effects, and destabilizing forces (that roughen the interface) that are induced by pinning effects of material heterogeneities. The analytical solution of Schmittbuhl et al. (2004) reproduced the observed scaling behavior of natural stylolites and demonstrated that the two scaling regimes (characterized by the two different Hurst exponents) correspond to two thermodynamic regimes that are dominated by either surface or elastic energies on small and large scales, respectively (Renard et al., 2004; Schmittbuhl et al., 2004, Gratier et al., 2005). Based on the work of Schmittbuhl et al., (2004) 
101 it was demonstrated for the first time by Ebner et al., (submitted) that the crossover-length of

102 natural stylolites, which should be a function of the stress during stylolite growth, can be used

103 to determine stress magnitudes and burial depth in sedimentary basins. The discrete numerical

104 simulation technique of Koehn et al. (2007) enabled to study the dynamics of the roughening

105 process through time revealing that the stylolite interface width $w$ (defined in detail below)

106 grows as a power law with time $\left(w \sim t^{\beta}\right)$ with a growth exponent $\beta$ of 0.5 in the surface energy

107 dominated regime and a growth exponent of 0.8 in the elastic energy dominated regime. In

108 addition the roughness growth may saturate so that the stylolites lose their memory for

109 compaction or finite strain. It is important to notice that the roughness of simulated stylolites

110 in this contribution is produced by heterogeneities in the material that pin the stylolitic

111 interface due to slower dissolution rate constants, which are in competition with the surface

112 and elastic energies which tend to flatten the surface (Koehn et al., 2007). Therefore the

113 obvious question to ask is whether a variation of the quenched noise changes the scaling

114 properties of the stylolitic interface?

115 Thus, in the present contribution we investigate the influence of different heterogeneities

116 (namely the percentage of pinning particles, their pinning factor (defined below), and their

117 size) on the scaling behavior, dynamic growth, and determined crossover length of simulated 118 stylolites.

\section{2. Setup numerical model}

121 The numerical technique that we use to simulate stylolite roughening is based on a lattice122 spring model coupled with a dissolution routine (Koehn et al., 2004, 2006, 2007). The model

123 itself is embedded as a module in the "Elle" modeling-platform (Bons et al., 2008).

124 For computational reasons, to access large systems and analyze scaling laws over a large ratio

125 between the system size and the resolution, we will consider situations spatially invariant 126 along one of the directions tangential to the stylolite - and effectively treat systems with two 
127 spatial dimensions. The initial configuration of this $2 \mathrm{D}$ model, as shown in Fig. 2a, contains a

128 predefined flat interface that is considered to be filled with a confined fluid. Two blocks of

129 particles are separated by a fluid pocket. Such an approximate configuration would be

130 expected for example, for a fluid pocket embedded between two lowly permeable

131 sedimentary layers. This model system represents two solids or rocks that are pressed together

132 by inward moving top and bottom boundaries, whereas the side boundaries remain fixed

133 (uniaxial strain). A quenched noise (denoted by darker particles in Fig $2 \mathrm{a} \& \mathrm{~b}$ ) is introduced

134 by a change of the dissolution rate constant of a certain fraction of the particles (= pinning

135 particles) and represents material heterogeneities initially present in the host rock of natural

136 stylolites.

2.1. Theory

This section provides only a cursory review on the governing equations of the dissolution

140 process used in the model, for a detailed description and implementation the reader is referred

141 to Koehn et al., (2007) and Bons et al., (2008).

142 The pressure solution process is discretized in steps of dissolution of entire particles,

143 following a linear rate law (Koehn et al., 2007 and references cited therein) according to

$$
D=k V\left[1-\exp \left(-\frac{\left\{\Delta \psi+\Delta \sigma_{n}\right\} V}{R T}\right)\right]
$$

145 where $D$ is the dissolution velocity of the interface $\left(\mathrm{m} \mathrm{s}^{-1}\right), k$ a dissolution kinetics rate 146 constant $\left(\mathrm{mol} \mathrm{m} \mathrm{m}^{-2} \mathrm{~s}^{-1}\right), V$ the molecular volume of the solid $\left(\mathrm{m}^{3} \mathrm{~mol}^{-1}\right), R$ the universal gas

147 constant $\left(8.314 \mathrm{~J} \mathrm{~mol}^{-1}{ }^{\circ} \mathrm{K}^{-1}\right), T$ the temperature $\left({ }^{\circ} \mathrm{K}\right), \Delta \psi(\mathrm{Pa})$ the changes in Helmholtz free

148 energy density (which accounts for the variations in elastic and surface energies) of the solid

149 during dissolution of a solid element, and $\Delta \sigma_{n}(\mathrm{~Pa})$ the differences between the average

150 normal stress along the interface and the local normal stress at a specific location which is due

151 to the repulsion of the solids(Koehn et al., 2007). 
152 Surface energies $\left(E^{S}\right)$ of particles are calculated from the local curvature of the interface

153 around each particle, which can be expressed as

$$
E^{s}=\frac{\gamma}{\rho}
$$

155 where $\gamma$ is the surface free energy and $\rho$ is the local radius of curvature of the interface. We 156 consider a plane strain situation, i.e. an invariance along the third spatial dimension, so one 157 radius along the $2 \mathrm{D}$ plane investigated entirely characterizes the curvature of the interface -

158 the radius of curvature along the direction of invariance is infinite, and no surface energy is 159 associated to this direction. The surface energies of individual particles are averaged over 160 their neighbors to avoid artifacts from the discreteness of the model (for details see Koehn et 161 al., 2007).

162 In the lattice spring model every particle $(i)$ is connected to its neighbors $(j)$ via a triangular 163 linear elastic spring network. The elastic energy $\left(E^{e l}\right)$ of a single element is given by

$$
E^{e l}=\frac{1}{4} \sum_{(j)} \kappa\left(\left|x_{i}-x_{j}\right|-l\right)^{2}
$$

165 where the sum is over all neighbors $(j), \kappa$ is a spring constant, $l$ is the equilibrium distance 166 between elements $i$ and $j$.

\subsection{Basic numerical step}

169 The constitutive equations stated above are implemented as follows:

170 - Top and bottom walls are moved inwards simultaneously at a given time/deformation $171 \quad$ step.

- For every deformation step the rate law (Eq. 1) is used to calculate if individual

173 particles at the interface can dissolve in the given time as soon as the two solids meet.

174 - When elements dissolve they are removed completely and the system can relax. 175 Relaxation is accomplished by an over-relaxation algorithm that finds the new 
equilibrium configuration for the lattice. Dissolution of particles can take place as long as the given time for the individual deformation step is not consumed.

- If the deformation time is used up or no particles can dissolve within the given time the system is stressed again by a deformation step.

\subsection{Parameters, boundary conditions, limitations}

182 The material parameters we use resemble those of a limestone, these values are analog to 183 those found in reference works (e.g. Clark, 1966) and in line with values used for the 184 analytical solutions of Renard et al. (2004) and Schmittbuhl et al. (2004): a molar volume of $0.00004 \mathrm{~m}^{3} / \mathrm{mol}$, a Young's Modulus of $80 \mathrm{GPa}$, a Poisson's ratio of 0.33 (this number is given by the triangular lattice configuration), a surface free energy of $0.27 \mathrm{~J} / \mathrm{m}^{2}$, a temperature of $300 \mathrm{~K}$ and a dissolution rate constant of $0.0001 \mathrm{~mol} /\left(\mathrm{m}^{2}-\mathrm{s}\right)$. In addition, the displacement rate of the upper and lower boundaries are fixed at a constant value corresponding to velocities between $10^{-10}$ and $10^{-12} \mathrm{~m} \mathrm{~s}^{-1}$.

190 The boundary condition can be seen as equivalent to a constant load boundary condition since

191 the dissolution process is fast enough to relax the stresses that build up during a single

192 deformation step. The sidewalls remain fixed during the model runs and there is no wrapping 193 of particles in the x-direction of the model.

194 We use three basic model setups for which we systematically vary the heterogeneities in the 195 structure. All boxes used have the same number of particles (400 particles) in the x-direction 196 but three different particles-sizes were used $0.01 \mathrm{~mm}, 0.1 \mathrm{~mm}$ and $1 \mathrm{~mm}$, which corresponds 197 to absolute box-sizes of 4, 40 and $400 \mathrm{~mm}$.

198 To introduce the quenched noise in the simulations a pseudorandom algorithm is used to 199 create a spatial Gaussian distribution of particles that dissolve slower (pinning particles). We 200 varied three parameters of the quenched noise in this study: (i) number of pinning particles in 201 a range from 1-20\%, (ii) dissolution rate constant of pinning particles (from 0.1-0.99 
normalized to the dissolution rate constant of the matrix, which is 1), which determines the

203 pinning factor and (iii) the absolute size of the heterogeneities which varies with the particle

204 size in the range of $0.01-1 \mathrm{~mm}$.

205

206

\section{Data analysis \& results}

207 The individual model runs are grouped with respect to the particle-size of the model and are

208 termed surface, intermediate and elastic class according to the dominance of the energy

209 regime during the roughening process. The surface class has a particle size of $0.01 \mathrm{~mm}$ (box

210 size of $4 \mathrm{~mm}$ ), the intermediate class a particle size of $0.1 \mathrm{~mm}$ (box size of $4 \mathrm{~cm}$ ) and the

211 elastic class a particle size of $1 \mathrm{~mm}$ (box size of $40 \mathrm{~cm}$ ). In nature the particles may resemble

212 actual grains so that the grain size of the rock varies between the different classes.

213 Unfortunately we are restricted by the resolution of the numerical model to 400 particles in

214 the $\mathrm{x}$-direction, with this resolution a single simulation may run from 10 to 15 days on 4 cores

215 of a recent workstation. Figure 3 shows the roughening of stylolites of these three classes,

216 each with identical quenched noise (5\% pinning particles with half the dissolution rate of the

217 matrix) to demonstrate the influence of the absolute box/particle size. The differences in the

218 roughness and the roughness growth can easily be seen when individual steps of different

219 classes are compared but also by following the growth of individual stylolite peaks along the

220 time axis. In the elastic class individual peaks are growing very persistently whereas in the

221 surface class the growth is often disrupted, due to dissolution of pinning particles as a result

222 of high surface energies along pronounced peaks.

223 In the following sections we concentrate on the influence of the noise (amount and pinning

224 factor of the noise particles) on 1) the roughness exponents, 2) the growth exponents and 3)

225 the crossover length.

226

227

\subsection{Roughness exponents}


228 To quantitatively characterize the roughness of an individual 1D profile of a stylolite we used 229 concepts from statistical physics (Barabasi \& Stanley, 1995), which are briefly introduced in 230 the first part of this section. The methods used here are the same as those of previous studies 231 of natural stylolites (Renard et al., 2004; Schmittbuhl et al., 2004; Gratier et al., 2005; Ebner 232 et al., submitted), which facilitates comparison.

233 The prerequisite for the application of these scaling methods is that the 1D signal of the 234 numerical stylolite obeys a self-affine scaling invariance, which is given by (e.g. Barabasi \& 235 Stanley, 1995)

$$
f(b x) \sim b^{\alpha} f(x)
$$

where $f(x)$ is a single valued function and the power-law exponent $\alpha$ is called roughness or Hurst exponent and provides a quantitative measurement of the roughness of the signal. A self-affine function must be rescaled differently in $\mathrm{x}$ and $\mathrm{y}$ directions to obtain a scaling invariance i.e. horizontal rescaling of the form $x \rightarrow b x, b$ being a dilation factor, has to be rescaled in the vertical direction by $y \rightarrow b^{-a} y$ to obtain a scaling invariance. Different statistical methods can be used to evaluate the self-affine character of a signal and to determine the

244 associated roughness exponent. We apply two independent methods in this contribution, the 245 Fourier method and the Average Wavelet Coefficient method.

246 The Fourier method (e.g. Barabasi \& Stanley, 1995; Schmittbuhl et al., 1995) is based on a 247 Fourier transform of the original 1D signal (Fig. 4a). For every 1D signal (every deformation 248 step) the Fourier power spectrum $P(k)$ i.e., the square of the modulus of the Fourier transform, 249 was calculated as a function of the wave-number $k$. Plotting $P(k)$ as a function of $k$ in $\log -\log$ 250 space reveals a linear trend for a self-affine function (Fig. 4b), and the slope is a function of 251 the Hurst exponent through (Renard et al., 2004; Schmittbuhl et al., 2004):

$$
P(k) \sim k^{-1-2 \alpha} .
$$


253 The Average wavelet coefficient method ( $A W C$ ) was used as a second independent method to

254 confirm the scaling results (Simonsen et al., 1998; Hansen et al., 2000). This method is again

255 based on a decomposition of the 1D signal into wavelets, whose amplitude depends on scale 256 and the position. The wavelet transform is defined after Simonsen et al. (1998) by

$$
W_{a, b}=\frac{1}{\sqrt{a}} \int_{-\infty}^{\infty} \psi\left(\frac{x-b}{a}\right) f(x) d x,
$$

258

259

260

261

where $\psi$ is the wavelet basis (Daubechies wavelet of order 12) which is parameterized by a scale parameter $a$ and a translation parameter $b$, and $f$ is the single-valued original function. Finally the wavelet coefficients are averaged over the translation parameter $b$ for every $a$ to obtain the average wavelet coefficient $W(a)$. If the input signal is self-affine, the wavelet transform verifies that the average wavelet coefficient $W(a)$ scales as (Simonsen et al., 1998)

$$
W(a) \sim a^{\alpha+1 / 2} .
$$

Plotting the average wavelet coefficients as a function of the scale parameter $a$ in $\log -\log$ space (Fig. 4c), the slope of the linear regression through the data is again a function of the Hurst exponent.

Using these two statistical methods, we first study the dynamics of the roughness exponents through time during stylolite growth, and then concentrate on their stability with respect to variations of the noise. The roughness exponents increase relatively quickly in the course of a simulation run (Fig. 4d) and become stable after model step 3000 with only minor fluctuations. The Fourier-as well as the $A W C$-method show consistent evolutions and similar values of the roughness exponents. Averages of the plateau values reached (after step 3000) for individual model runs are used as a characteristic value for the roughness exponent for a specific setup. Error bars underline the standard deviation around this average (Fig. 5).

The surface class is characterized by consistently high values for the Hurst exponent i.e. $\alpha \sim 1$ 1.1, independent of the pinning factor (i.e. the dissolution rate constant $k$ in Eq 1; cp Fig. 5a) or the amount of pinning particles (Fig. 5b). 
278 The exponents only decrease when the pinning factor of particles is very low (dissolution rate 279 constant $>0.9$ ) and the rock becomes very homogeneous. The most stable roughness 280 exponents for the surface class are reached in the range 0.1 to 0.8 for the pinning factor and 1 281 to $20 \%$ of pinning particles.

282 The elastic class reveals lower Hurst exponents ( $\alpha \sim 0.6$ to 0.9$)$ than the surface class. If the 283 pinning factor of particles is very strong (below the value 0.4 for the relative dissolution rate 284 constant in Fig. 5a) stress concentrations are locally too high once two pinning particles meet 285 and artifacts develop (usually anticracks that grow laterally emerge from these concentrations, 286 hence modifying the surface topography) in the numerical model within the elastic class. 287 Therefore we did not include values below 0.4 from elastic simulations in Fig. 5a. Generally 288 the roughness exponents in the elastic class show stronger fluctuations than those of the 289 surface class. They are relatively stable within a pinning factor range of 0.5 to 0.8 (Fig. 5a) 290 and 1 to $20 \%$ of pinning particles.

291 The surface and elastic classes correspond well to the two scaling regimes found in natural 292 stylolites (Renard et al., 2004; Schmittbuhl et al., 2004) that are separated by a crossover293 length at the millimeter scale. The roughness exponents of the surface class $(\alpha \sim 1.1)$ are in 294 very good agreement with analytical predictions and experimental observations (e.g. Gratier 295 et al., 2005). The elastic class displays values for the Hurst exponent $(\alpha \sim 0.6-0.9)$ that are 296 higher than exponents from natural examples (Renard et al., 2004; Schmittbuhl et al., 2004; 297 Ebner et al., submitted) or analytical predictions, which are generally around 0.5. These 298 analytical predictions are usually based on linear approximations, which are strictly speaking 299 valid as long as the surface morphology is not too developed. The present model does not 300 present any such limitations, and the fully developed situation can thus present a different 301 Hurst exponent from the initial one. The discrepancy with natural data may arise from the 302 large particle (or grain) size that we use in the setup for the elastic class. In nature the grain 303 size is much smaller and corresponds to the values that we use in the surface class. 
306 In addition to the dynamic development of the roughness exponents the simulations allow us 307 to study how fast the amplitude of the stylolite roughness grows through (model-) time or as a 308 function of the finite strain. First we concentrate on different growth regimes of stylolites, the 309 associated growth exponents and prefactors of scaling functions and then study the variation 310 of these factors as a function of host-rock heterogeneities. In order to quantify the amplitude 311 of the roughness we use the interface width $(w)$ that is defined as the root-mean-square 312 fluctuation of the height of the interface for a given time-step (Barabasi \& Stanley, 1995)

$$
w(L, t) \equiv \sqrt{\frac{1}{L} \sum_{i=1}^{L}[h(i, t)-\bar{h}(t)]^{2}},
$$

where $w$ is the interface width as a function of system size $L$ and time $t, h$ is the height of 315 point $i$ on the interface at time $t$ and $\bar{h}$ the average height of the interface at time $t$ given by

$$
\bar{h}(t) \equiv \frac{1}{L} \sum_{i=1}^{L} h(i, t)
$$

317 In our simulations, the system size $L$ is defined as the number of elements in the x-direction, 318 which is constant for all simulations, i. e. 400 particles. Roughening processes of interfaces in 319 a wide range of fields have been demonstrated to follow a power law in time (e.g. Barabasi \& 320 Stanley, 1995) defined by a growth-exponent $\beta$ (given by, $w(L, t) \sim t^{\beta}$ ). This initial phase of interface growth is usually followed by a second regime during which the interface width reaches a saturation value, $w_{\text {sat }}$, which is directly related to the system-size.

Both, the growth and saturation regimes can be seen in Fig. 6a for an experiment of the 324 surface class with a characteristic growth exponent of $\beta \sim 0.5$. The arrow in Figure 6a marks 325 the transition from the power law growth regime to the regime where the interface width 326 saturates and stays constant. The intermediate class simulations show a similar growth 327 exponent (around 0.5) but do not saturate in the given deformation time. The elastic class 
328 (Fig. 6b) shows two successive growth regimes, the first being defined by a growth exponent 329 of $\beta \sim 0.5$ up to a crossover interface width followed by a second regime with $\beta \sim 0.8$ without 330 reaching the saturation regime. We suggest the following schematic growth regimes (Fig 6c)

331 for stylolites: (i) growth in the surface energy dominated regime with an exponential growth 332 defined by $\beta \sim 0.5$ followed by (ii) growth in the elastic energy dominated regime with $\beta \sim 0.8$ 333 and finally reaching (iii) a saturation regime where the interface width stays constant.

334 This strict non-linearity of the interface growth in our simulations suggests that estimated 335 amounts of compaction (here used as synonymous with volume/area loss due to pressure 336 solution) from stylolite amplitude heights (e.g. Tada \& Siever, 1989 and references cited 337 therein) only capture a small part of the actual compaction. To cope with this problem, Koehn 338 et al. (2007) demonstrated that the actual displacement can be expressed for the elastic or 339 surface energy dominated growth regimes as a function of the interface width and the growth 340 exponent (as long as the critical saturation time is not reached), given by

$$
A \sim(w / l)^{1 / \beta} l
$$

342 where, $A$ is the compaction displacement, $w$ the interface width, $\beta$ the growth exponent (for a 343 certain class) and $l$ the particle size. The slope of this function gives a prefactor for the scaling 344 relation which should remain constant until the saturation time is reached. We call the slope 345 of this relation here and in the subsequent sections compaction prefactor because it relates the 346 interface width to the total compaction (Fig. 7). As soon as the saturation time is reached the 347 relation does not hold any more and the function deviates from the linear trend (Fig. 7a). This 348 effect can be observed in Figure $7 \mathrm{a}$ where the arrow marks the onset of the saturation of the 349 interface width, compaction goes on but the interface width (x-axis) remains constant. 350 However, if the saturation is not attained (Fig. 7b) the actual compaction can be calculated 351 accurately from the interface width and the growth exponent using Eq. 9.

352 Looking at the growth exponent as a function of the quenched noise it can be clearly 353 demonstrated that neither a variation in the pinning factor of particles (Fig. 8a) nor in the 
354 amount of pinning particles (Fig. 8b) has a significant influence. The values for the growth 355 exponent cluster around $\beta \sim 0.5$ for the surface and intermediate classes and around $\beta \sim 0.8$ for 356 the elastic class. The compaction prefactors display a higher variability than the growth 357 exponents but no systematic trend can be seen that relates this variation to the pinning factor 358 or amount of pinning particles in the host-rock. Figures $8 \mathrm{c} \& 8 \mathrm{~d}$ show that there is no 359 significant difference between the three classes of particle sizes used with values for the 360 compaction prefactor in a range between $\sim 12$ and $\sim 25$. As a third quantity we compare the 361 maximum interface width normalized by the particle size $\left(w_{\max }\right)$ that develops during 362 simulations with different heterogeneities (Fig. 8e \& f). The largest interface widths are 363 achieved in the elastic class with $w_{\max } \sim 20$ in contrast to $w_{\max } \sim 10$ reached in the intermediate 364 and surface class. Hence the interface growth displays twice the displacement in the elastic 365 class in the given simulation time due to the larger growth exponents than those of the 366 intermediate or surface class, respectively. For the surface, intermediate and elastic classes 367 the variation of the pinning factor of particles (Fig. 8e) have no considerable influence on the 368 maximum interface width. However the amount of pinning particles has a significant 369 influence on the surface and intermediate class (Fig. 8f). Both classes show an evident 370 decrease in the maximum interface width with increasing amount of pinning particles. This 371 trend cannot be observed in the elastic class (Fig. 8f).

372 We also tested the influence of the initial shape of the predefined interface separating the two 373 blocks that are pressed together during an experimental run (compare Fig. 2a), which is flat in 374 all the simulation data shown in the preceding sections. To investigate the dynamic roughness 375 evolution of an already rough interface we arbitrarily choose a time/deformation step $\left(t_{n}\right)$ of a 376 simulation run (Fig 9a) and subtracted the topography $(h)$ of this step from the subsequent 377 time steps similar to $h(t)=h\left(t_{n}+m \cdot \Delta t\right)-h\left(t_{n}\right)$. This procedure allows to investigate the dynamic 378 evolution of a rough interface but statistically evaluating the difference of the evolving 379 roughness from time $t_{n}$ onwards (Fig 9b). Departing from an already rough interface does not 
change the scaling parameters (growth and roughness exponent) as depicted in Fig 9. Hence

381 the model setup we choose in this work (i.e. a flat initial interface) can also account for 382 complex initial topographies.

383

\subsection{Crossover length scales}

385

$402 w(x)=\frac{(\tanh (x+L)+1)}{2}$, submitted)

The crossover-length of the stylolite roughness that separates the surface energy regime from the elastic energy dominated regime is a function of the stress during stylolite growth and can be used as paleo-stress gauge. It is of fundamental importance to know if this crossover is constant when the heterogeneities in the host-rock vary. In the following section we want to explore how sensitive the crossover-length scale is on variations in the amount and strength of pinning particles. The surface and elastic classes of our simulations reveal roughness exponents that are characteristic for the two regimes where surface or elastic energy are dominant. The crossover-length between the two regimes can be found in the intermediate class of our simulations that reveals two different roughness exponents and hence the transition between the two scaling regimes (Fig. 10). The Fourier power spectrum of the 1D signal of a stylolite in the intermediate class (Fig. 10b) shows a change from a shallow to a steep slope, i.e. small and large roughness exponents on large and small scales, respectively. To avoid bias due to improper fitting of the crossover-length we used a nonlinear least square curve fitting algorithm in logarithmic space to model our scaling function (Ebner et al.,

$f(x)=\left(a_{L} x+m_{L}\right)(1-w(x))+\left(a_{S} x+m_{S}\right) w(x)$

403 where $a_{L, S}$ are the exponents of the scaling function for large and small scales, $m_{L, S}$ the 404 corresponding intercepts with the ordinate and $w(x)$ the weighting function. During this 
405 procedure the roughness exponents of our nonlinear model function were fixed according to

406 the roughness exponents $\left(\alpha_{S} \sim 1.1 ; \alpha_{L} \sim 0.5\right)$ reported from natural stylolites (Renard et al., 2004;

407 Schmittbuhl et al., 2004, Ebner et al., submitted).

408 The crossover-lengths obtained for all experiments of the intermediate class are in a range of

$409 L \sim 1.33 \pm 0.09 \mathrm{~mm}$, the crossover length usually develops simultaneously with the achievement

410 of the plateau values (compare Fig. 4d). Neither of the quenched noise parameters varied in

411 the experiments influences the crossover-length (Fig. 11).

412 The time evolution of the roughness presented in the previous section showed that the surface

413 energy dominated regime is characterized by a growth exponent of 0.5 whereas the elastic

414 energy dominated regime is characterized by a growth exponent of 0.8 . Looking at the

415 roughness growth in the elastic class one could still see the slow surface energy dominated

416 growth in the beginning of the roughness evolution (Fig. 6). Therefore the growth exponents

417 also show a transition (that we term the crossover interface width) between growth in the

418 surface energy dominated regime and growth in the elastic energy dominated regime, similar

419 to the two roughness exponents that are characteristic for these two regimes. The crossover

420 interface width is very consistent for all experiments with $w \sim 1.23 \pm 0.04 \mathrm{~mm}$, independent of

421 the quenched noise introduced in the system. We did not observe a crossover in the interface

422 growth of the intermediate and surface class because the interface width in these classes is

423 simply not large enough to reach the elastic growth regime. Due to the very good correlation

424 between the magnitudes of the crossover-length and the crossover interface width (Fig. 11)

425 we argue that both crossovers arise from the same process, namely the transition from a 426 surface energy to an elastic energy dominated regime.

428 4. Discussion

429 In the following section we first discuss the influence of the quenched noise on the scaling 430 parameters in our numerical simulations, deal with the relevance of the noise and compare the 
results to natural stylolites. Secondly, we will focus on the crossover phenomena and their

432 significance for the estimate of volume loss along a finite natural stylolite.

433 The influence of the exact nature of the heterogeneities (i.e. pinning factor, amount and size 434 of pinning particles) on the scaling exponents can be directly investigated by a close 435 examination of Figures 5, 8 and 11. Doing so, it is immediately evident that the pinning factor 436 has the least influence on the roughness and growth exponents as well as on the crossover 437 scaling ( $L$ and $w_{\text {cross }}$ ), with resulting values that are very consistent over wide ranges of the 438 parameter space. The amount of pinning particles only shows an influence on the maximum 439 interface width $w_{\max }$, which decreases with increasing amount of heterogeneities (Fig. 8f).

440 This fact is in good agreement with observations made on natural stylolites that stylolite 441 amplitudes decrease with the amount of heterogeneities i.e. clay particles (e.g. Tada \& Siever, 4421989 , and references cited therein). The scaling exponents themselves are independent of the 443 amount of pinning particles. The biggest influence exists between the different experiment 444 classes i.e. particle sizes, which reveal different roughness and growth exponents that are on 445 the other hand very consistent within an individual class. But it has been demonstrated 446 (Renard et al., 2004; Schmittbuhl et al., 2004, Koehn et al., 2007), that these differences arise 447 from a transition from a surface energy dominated regime (where surface energies smoothen 448 the interface) to an elastic energy dominated regime (where elastic energies smoothen the 449 interface). The differences in the scaling exponents between the experimental classes are 450 therefore not caused by the heterogeneities themselves but by the processes that govern the 451 roughening on respective scales. We are thus convinced that the influence of the exact nature 452 of the heterogeneities plays a minor role and that roughening is dominated by an inherent 453 process that depends on the length-scale.

454 The quenched noise we introduced in the different simulations, i.e. changes in the dissolution 455 rate constant that influence the dissolution velocity of a particle (cp. Eq. 1), is a simple 456 chemical noise as pointed out by Koehn et al. (2007). We are aware that along natural 
stylolitic interfaces the elastic parameters, surface energies, crystallographic orientations are

458 very likely to change, in addition to chemical variations. However the effects of a change of

459 these other parameters in our model also result in a change of the dissolution velocity. We

460 therefore argue that for the developing structure it should make no difference what the exact

461 nature of the noise is, since any particle with a slower dissolution velocity will pin the surface

462 and therefore cause a roughening of the interface.

463 It was demonstrated that individual natural stylolites from different outcrop localities and

464 lithologies, i.e. different host-rock compositions, reveal the same scaling behavior (e.g.

465 Renard et al., 2004; Schmittbuhl et al., 2004, Ebner et al., submitted). Consequently the 466 investigation of natural stylolites corroborates the fact that a common underlying mechanism

467 for stylolite roughening can be assumed rather than a roughening that is dominated by the 468 composition of the host rock.

469 Nevertheless we do not claim that knowledge of the exact nature or distribution of the 470 material heterogeneities is unimportant. Brouste et al., (2007) have shown that a changing 471 amount of heterogeneities might cause a stylolite to become a non-stationary signal with 472 alternating wavy and flat portions along the interface. We have not investigated the effects of 473 irregularly distributed noise since the heterogeneities are distributed equally in our model 474 setup.

475 The roughness data of simulated stylolites presented in this study reveal two self-affine 476 scaling regimes that are separated by a distinct crossover-length of $L \sim 1.3 \mathrm{~mm}$, which is well 477 in line with investigations of natural stylolites (Renard et al., 2004; Schmittbuhl et al., 2004, 478 Ebner et al., submitted). Additionally we have detected a crossover in the growth of the 479 interface during which the initial growth exponent of $\beta \sim 0.5$ up to a crossover interface width 480 of $w \sim 1.23 \mathrm{~mm}$ is replaced by a growth regime with an exponent of $\beta \sim 0.8$. Due to the very 481 good correlation between the magnitudes of the crossover-length and the crossover interface 482 width we argue that both crossovers arise from the same process, namely the transitions from 
483 a surface energy to an elastic energy dominated regime. The knowledge of the crossover

484 length $L$ which can be derived from the finite pattern of a natural stylolite with the above

485 methods is thus equivalent to knowledge of the crossover interface width. This fact has

486 important consequences regarding the assessment of the amount of total compaction of

487 individual stylolites. Substituting the growth exponents and the compaction prefactors found

488 for the two growth regimes in combination with the crossover-length, which separates the two

489 growth regimes (cp. Fig. 10c), into Eq. 10 should allow an exact reconstruction of the amount

490 of total compaction from finite pattern of a natural stylolites.

\section{5. Conclusions}

493 In the course of this contribution we evaluated the scaling properties of simulated stylolites,

494 which facilitate a quantitative comparison with natural examples, exactly reproducing their

495 scaling. We observed only minor correlation between the exact nature of the noise introduced

496 in the model or the topography of the predefined interface and the scaling parameters

497 investigated, concluding that inherent processes i.e. the transition from a surface to an elastic

498 energy dominated regime control the roughening process.

499 Nevertheless the amount of heterogeneities has a negative effect on the maximum interface

500 width $\left(w_{\max }\right)$ achieved during deformation revealing increasing interface width with

501 decreasing amount of quenched noise. The absolute particle/noise size influences the

502 roughness and growth exponents, which in turn is caused by the transition from a surface to

503 an elastic energy dominated regime. Therefore it is important to know how large the noise or

504 pinning particles are in natural systems. The transition from surface energy as the dominant

505 stabilizing force of the interface to the dominance of elastic energies is the causation for the

506 most significant scaling transitions: (i) the roughness is characterized by two distinct spatial

507 scaling regimes on small and large length scales, respectively; (ii) the interface growth reveals

508 two growth regimes with a growth exponent of $\beta \sim 0.5$ up to a crossover interface width that 
coincides with the crossover-length $L$ followed by a growth regime with an exponent of $\beta \sim 0.8$

510 that eventually saturates due to finite size effects; (iii) the crossover interface width $w$

511 coincides with the crossover-length $L$ and thus allows to accurately reconstruct the

512 compaction history of finite stylolite patterns.

513 Our study corroborates the evidence that the simple mechanisms summarized above support

514 analytical predictions and natural observations given in previous studies, and are a convincing

515 causation for the formation of stylolite roughness. However a detailed study on the exact

516 nature and distribution of quenched noise in the host rocks of natural stylolites would shed

517 light on the origin and initiation of these complex structures.

518

Acknowledgements

520

521

522

523

524

525

526

527

528

529

530

531

532

533

534

535

536

537

538

539

540

541

542

543

We are indebted to Jean Schmittbuhl for stimulating discussion. M. Ebner and D. Koehn acknowledge financial support through the DFG project KO2114/5-1, the MWFZ of Mainz and the Geocycles Cluster funded by the state of Rhineland-Palatinate.

\section{References}

Barabasi, A. L. \& Stanley, H. E. 1995. Fractal concepts in surface growth. Cambridge University Press.

Bathurst, R. G. C. 1987. Diagenetically Enhanced Bedding in Argillaceous Platform Limestones - Stratified Cementation and Selective Compaction. Sedimentology 34(5), 749-778.

Bons, P. D. D., Koehn, D. \& Jessell, M. W. 2008. Microdynamics Simulation. In: Lecture Notes in Earth Sciences (edited by Friedmann, G. M. \& Seilacher, A.) 106. Springer, Berlin, 406.

Brouste, A., Renard, F., Gratier, J. P. \& Schmittbuhl, J. 2007. Variety of stylolites' morphologies and statistical characterization of the amount of heterogeneities in the rock. Journal of Structural Geology 29(3), 422-434.

Buxton, T. M. \& Sibley, D. F. 1981. Pressure Solution Features in a Shallow Buried Limestone. Journal of Sedimentary Petrology 51(1), 19-26.

Clark, S. P. J. 1966. Handbook of Physical Constants. Geological Society of America, New York.

Dunnington, H. V. 1954. Stylolite development post-dates rock induration. Journal of sedimentary Petrology 24(1), 27-49.

Ebner, M., Koehn, D., Toussaint, R., Renard, F. \& Schmittbuhl, J. submitted. Scaling of natural stylolites and their use as stress-depth gauges. Geophysical Research Letters. 
Fletcher, R. C. \& Pollard, D. D. 1981. Anti-Crack Model for Pressure Solution Surfaces. Geology 9(9), 419-424.

Gratier, J. P., Muquet, L., Hassani, R. \& Renard, F. 2005. Experimental microstylolites in quartz and modeled application to natural stylolitic structures. Journal of Structural Geology 27(1), 89-100.

Guzzetta, G. 1984. Kinematics of Stylolite Formation and Physics of the Pressure-Solution Process. Tectonophysics 101(3-4), 383-394.

Hansen, A., Schmittbuhl, J., Batrouni, G. G. \& de Oliveira, F. A. 2000. Normal stress distribution of rough surfaces in contact. Geophysical Research Letters 27(22), 36393642 .

Heald, M. T. 1955. Stylolites in Sandstones. Journal of Geology 63(2), 101-114.

Katsman, R., Aharonov, E. \& Scher, H. 2006. A numerical study on localized volume reduction in elastic media: Some insights on the mechanics of anticracks. Journal of Geophysical Research-Solid Earth 111(B3), B03204.

Karcz, Z. \& Scholz, C. H. 2003. The fractal geometry of some stylolites from the Calcare Massiccio Formation, Italy. Journal of Structural Geology 25(8), 1301-1316.

Koehn, D., Dysthe, D. K. \& Jamtveit, B. 2004. Transient dissolution patterns on stressed crystal surfaces. Geochimica et Cosmochimica Acta 68(16), 3317-3325.

Koehn, D., Malthe-Sorenssen, A. \& Passchier, C. W. 2006. The structure of reactive grainboundaries under stress containing confined fluids. Chemical Geology 230(3-4), 207219.

Koehn, D., Renard, F., Toussaint, R. \& Passchier, C. W. 2007. Growth of stylolite teeth patterns depending on normal stress and finite compaction. Earth and Planetary Science Letters 257(3-4), 582-595.

Koehn, D., Ebner, M., Renard, F.,Toussaint, R. \& Passchier C. W. in preparation. The use of stylolites as paleo-stress-gauges.

Merino, E. 1992. Self-organization in stylolites. American Scientist 80(5), 466.

Merino, E., Calas, A. \& Fletcher, R. C. 2006. Genesis of self-organized zebra textures in burial dolomites: Displacive veins, induced stress, and dolomitization. Geologica Acta 4(3), 383-393.

Park, W. C. \& Schot, E. H. 1968. Stylolites: their nature and origin. Journal of sedimentary Petrology 38(1), 175-191.

Railsback, L. B. 1993. Lithologic Controls on Morphology of Pressure-Dissolution Surfaces (Stylolites and Dissolution Seams) in Paleozoic Carbonate Rocks from the Mideastern United-States. Journal of Sedimentary Petrology 63(3), 513-522.

Railsback, L. B. 1998. Evaluation of spacing of stylolites and its implications for selforganizations of pressure dissolution. Journal of Sedimentary Research 68(1), 2-7.

Renard, F., Schmittbuhl, J., Gratier, J. P., Meakin, P. \& Merino, E. 2004. Three-dimensional roughness of stylolites in limestones. Journal of Geophysical Research-Solid Earth 109(B3), -.

Rutter, E. H. 1983. Pressure solution in nature, theory and experiment. Journal of the Geological Society of London 140(5), 725-740.

Schmittbuhl, J., Vilotte, J. P. \& Roux, S. 1995. Reliability of Self-Affine Measurements. Physical Review E 51(1), 131-147.

Schmittbuhl, J., Renard, F., Gratier, J. P. \& Toussaint, R. 2004. Roughness of stylolites: Implications of 3D high resolution topography measurements. Physical Review Letters 93(23), -.

Simonsen, I., Hansen, A. \& Nes, O. M. 1998. Determination of the Hurst exponent by use of wavelet transforms. Physical Review E 58(3), 2779-2787.

Stockdale, P. B. 1922. Stylolites: their nature and origin. Indiana University Studies 9, 1-97. 
594

595

596

597

598

599

600

601

602

603

604

605

606

607

608

609

610

611

612

613

614

615

616

617

618

619

620

Tada, R. \& Siever, R. 1989. Pressure Solution during Diagenesis. Annual Review of Earth and Planetary Sciences 17, 89-118.

\section{Figure captions}

Figure 1: Plane section of a bedding parallel stylolite in a jurassic limestone from Cirque de Navacelles (southern France). The rough interface is accentuated by a thin clay layer that is considered to be the residuum of the dissolved rock mass.

Figure 2: Simplified sketch of the setup of the numerical model (modified after Koehn et al., 2007).The top and bottom walls of the box are moved inwards simultaneously to stress the system and initiate dissolution along the interface. a) Initial configuration of the setup showing a flat interface (dashed line). b) Configuration after a certain amount of compaction. The interface (dashed line) has developed a distinct roughness, note that the heterogeneities (darker spheres) accumulate along the interface.

Figure 3: Growth of three stylolites with similar heterogeneities but different lengths and discretization resolution. Left panels show 3D plots of the stylolite growth from a flat interface until the end of the experiment. Right panels show 3 individual deformation steps (step 400, $2000 \& 4000$ ) corresponding to the solid lines in the 3D plot a) surface class (box size $=0.4 \mathrm{~cm})$ b) intermediate class (box size $=4 \mathrm{~cm})$ c) elastic class (box size $=40 \mathrm{~cm})$; Notice the disrupted growth of the surface class whereas the elastic class exhibits very continuous growth and pronounced peaks and teeth.

Figure 4: Roughness characterization of single deformation steps by means of the Hurst exponent. a) $1 \mathrm{D}$ profile (deformation step 3000) of the surface class with $5 \%$ pinning particles and a dissolution rate constant of $k=0.4$; b) Fourier power spectrum $P(k)$ of the signal from 
621 Fig. 4a plotted as a function of the wavenumber $k$. Linear regression (solid line) is calculated 622 from the binned spectra (crosses), the slope of the regression is a function of the Hurst 623 exponent, see section 3.1.1. for detailed description. c) Wavelet spectra $W(a)$ plotted (crosses) 624 as a function of the scaling parameter $a$ (see average wavelet coefficient-method in section 625 3.1.1.). The slope of the linear regression (solid line) is again a function of the Hurst 626 exponent. d) Evolution of the Hurst exponent in the course of an entire experiment for the 627 Fourier and AWC methods. Stars indicate the Hurst exponent of the 1D signal shown in Fig. $6284 \mathrm{a}$ for the two methods used. Notice the leveling off at a specific Hurst exponent (plateau 629 value) which is characteristic for all simulations.

632 Figure 5: Composite plots showing the roughness data (Hurst exponents) for the surface and 633 elastic classes. a) Hurst exponent plotted versus dissolution rate constants $k$ with a fixed 634 amount of pinning particles of $5 \%$ (where $k=1$ is the dissolution rate of the matrix). b) Hurst 635 exponent plotted versus amount of pinning particles with a dissolution rate constant of $k=0.5$ 636 for all experiments. The error bars correspond to the standard deviation given by the 637 fluctuation around the plateau values, compare Fig 4d.

638

639 Figure 6: Interface growth depicted by means of the interface width (Eq. 8). a) Log-log plot 640 of the interface width as a function of time in the surface class (5\% pinning particles \& 641 dissolution rate constant of 0.4 ). Growth exponent $\beta=0.5$ is given by the slope of the linear 642 regression (solid line). Notice the saturation point (indicated by arrow) i.e. interface width 643 remains constant during ongoing deformation. b) Log-log plot of the interface width as a 644 function of time in the elastic class (5\% pinning particles \& dissolution rate constant of 0.4 ).

645 Notice the two successive growth regimes characterized by an initial growth exponent of $646 \beta=0.5$ up to a crossover width (indicated by arrow) followed by an exponent of $\beta=0.8$; no 
647 saturation can be observed within the given simulation time. c) Proposed composite graph of 648 the interface growth of simulated stylolites. Two successive growth regimes separated by a 649 narrow crossover width that are dominated either by surface or elastic energies are followed 650 by a saturation of the interface growth due correlation introduced by finite size effects.

651

652 Figure 7: Total compaction displacement expressed as a linear function (solid line) of the 653 interface width and the compaction prefactor (compare Eq. 10). a) Surface class experiment $654(5 \%$ pinning particles \& dissolution rate constant of $k=0.4)$ demonstrates that the relationship 655 is only valid as long as the interface is not saturated i.e. strong deviation from linear trend 656 (compare Fig 6a). b) Elastic class experiment (5\% pinning particles \& dissolution rate 657 constant of 0.4 ) reveals an accurate reproduction of the linear relationship (solid line) stated in 658 Eq. 10. No saturation was observed in the given simulation time.

659

660 Figure 8: Cumulative data for the surface, intermediate and elastic classes for: a) Growth 661 exponent versus dissolution rate constant (pinning particles fixed at 5\%); b) Growth exponent 662 versus amount of pinning particles (dissolution rate constant fixed at $k=0.5$ ); c) Compaction 663 prefactor versus dissolution rate constant (pinning particles fixed at 5\%); d) Compaction 664 prefactor versus amount of pinning particles (dissolution rate constant fixed at $k=0.5$ ). 665 Maximum interface width normalized by the particle size attained during experimental runs 666 for the surface, intermediate and elastic class e) with changing dissolution rate constant (with $6675 \%$ pinning particles); f) with changing amount of pinning particles (dissolution rate constant 668 is fixed at 0.5).

669

670 Figure 9: The influence of the topography of the initial predefined interface on the dynamic 671 roughness evolution. a) Rough initial interface used as starting point to evaluate the interface 672 width evolution on top of this roughness for a surface class simulation with $5 \%$ pinning 
673 particles and a pinning factor of 0.5 (for details see text). b) Comparison of the interface

674 width evolution of an initially flat and rough (see Fig 9a) interface. Both interfaces show a 675 similar evolution with growth exponents of 0.54 and 0.51 for the flat and rough initial 676 interface respectively. Notice that both growth and the roughness exponents (not shown) are 677 independent of the initial topography of the predefined interface.

678

679 Figure 10: Crossover phenomena in the roughness scaling and interface growth. a) 1D signal 680 of an experiment from the intermediate class (5\% pinning particles; dissolution rate constant $681 k=0.4$ ); b) Fourier power spectrum (inset) of the signal and binned spectra (crosses). A 682 nonlinear model function (for explanation see text) used to minimize the original data (solid 683 line) is used to accurately locate the position of the crossover length (triangle) $L=1.27 \mathrm{~mm}$; c) 684 Log-log plot of the interface width versus time of the elastic class $(5 \%$ pinning particles \& 685 dissolution rate constant $k=0.4$ ). Notice the two successive growth regimes characterized by 686 an initial growth exponent of $\beta=0.5$ up to a crossover width $w=1.24 \mathrm{~mm}$ followed by an 687 exponent of $\beta=0.8$. Notice that both crossover scales correspond to the transition from a 688 surface to an elastic energy dominated regime.

689

690 Figure 11: Crossover length $L$ and crossover interface width $w$ plotted as a function of the 691 quenched noise. a) Crossover length (circles) calculated from the Fourier power spectrum (cp. 692 Fig. 10b) and crossover interface width (diamonds) for simulations with different dissolution 693 rate constants. b) Crossover length (circles) and crossover interface width (diamonds) for 694 simulations with different amounts of pinning particles.

695 
Click here to download high resolution image

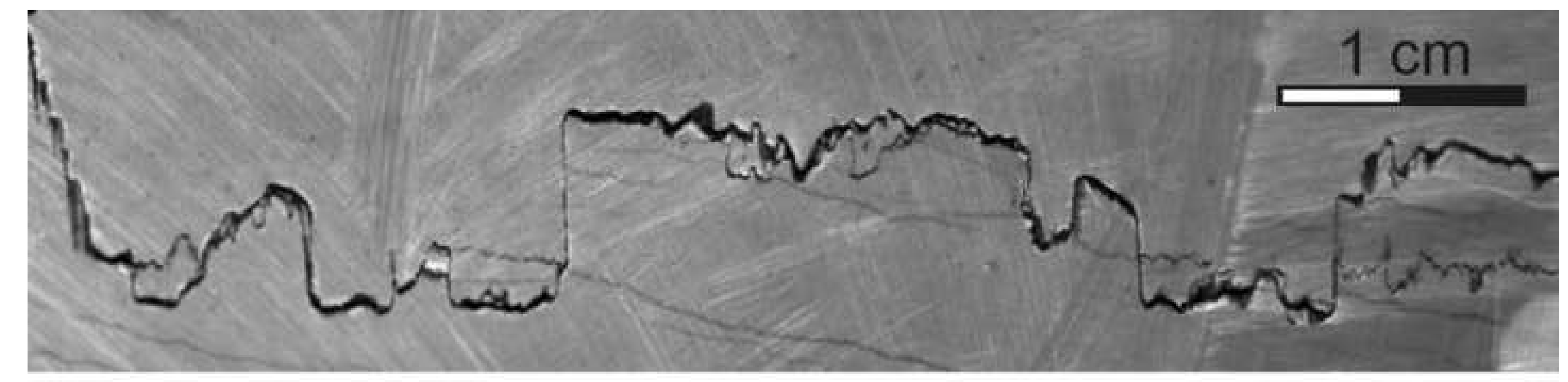

Click here to download high resolution image

$$
\text { Figure } 1
$$

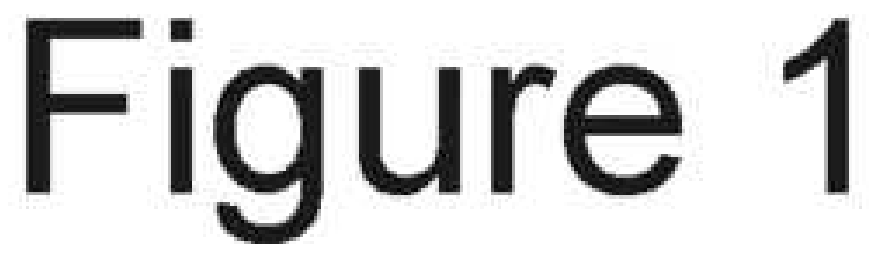

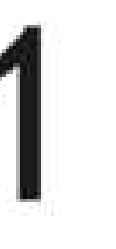


a

b

constant deformation rate

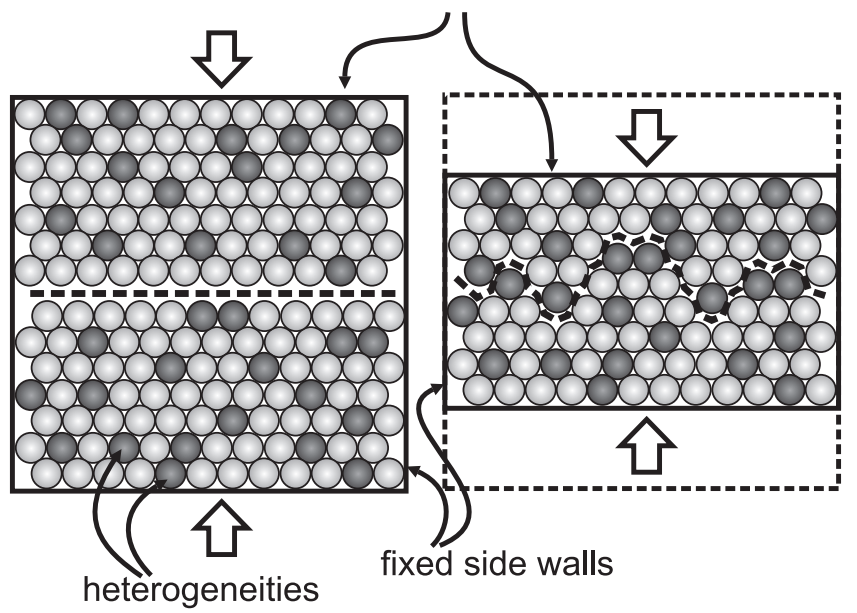

Figure 2 heterogeneities 
Figure 3
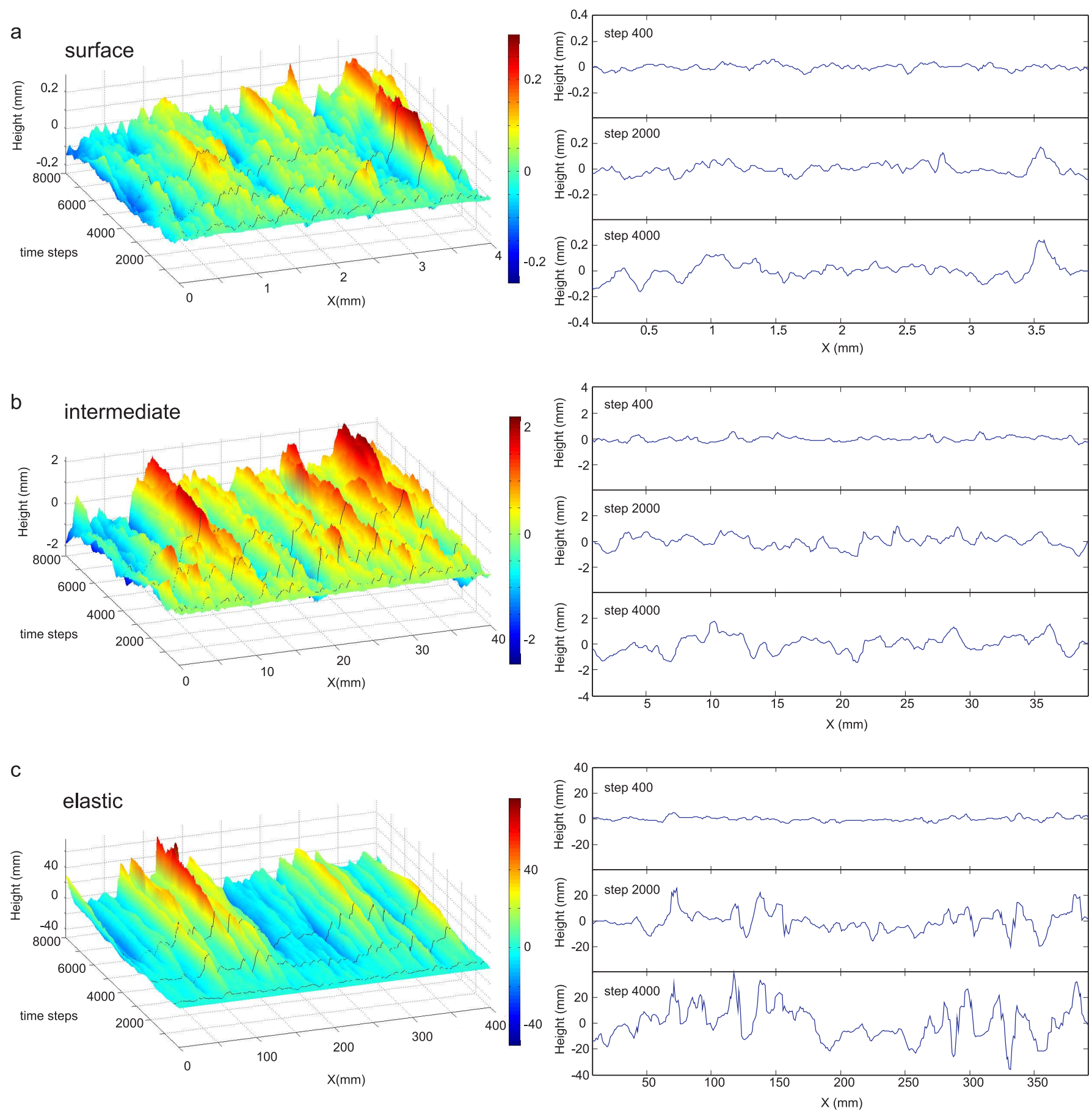

Figure 3 
Figure 4

a
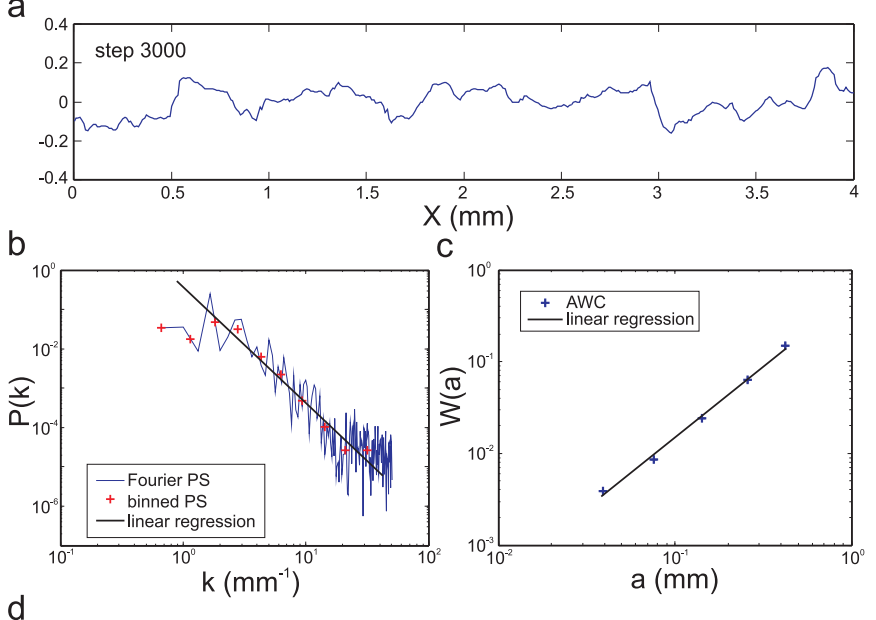

$c$

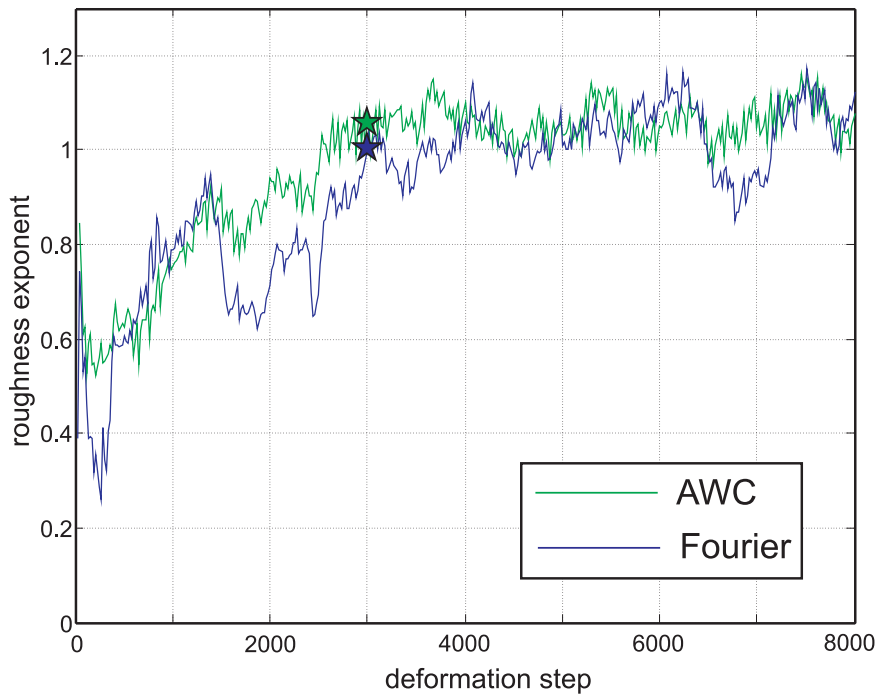

Figure 4 


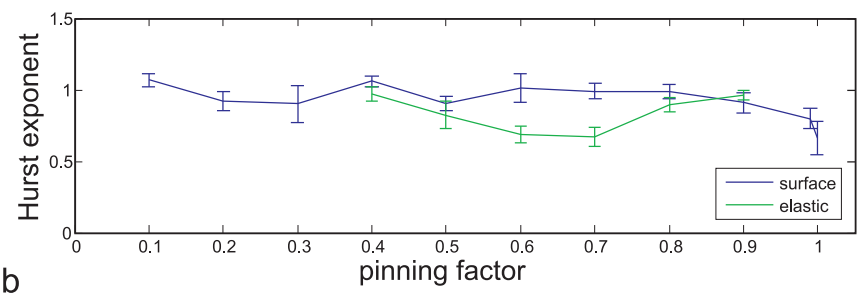

b

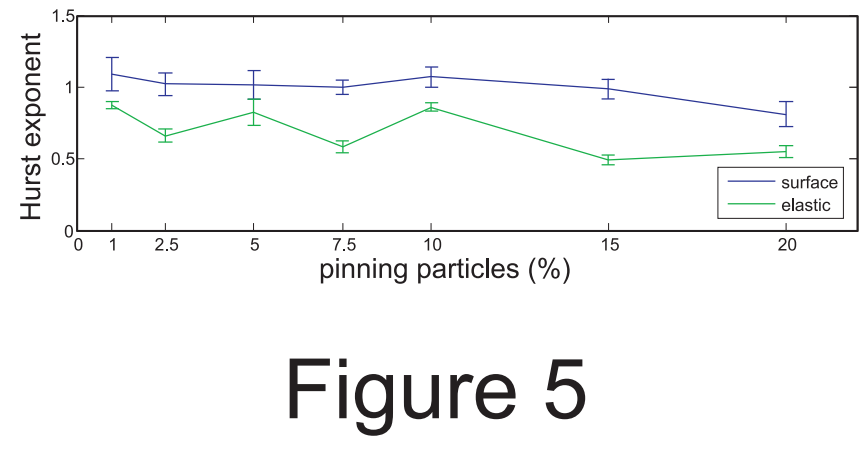

Figure 5

pinning particles (\%) 
Figure 6

b

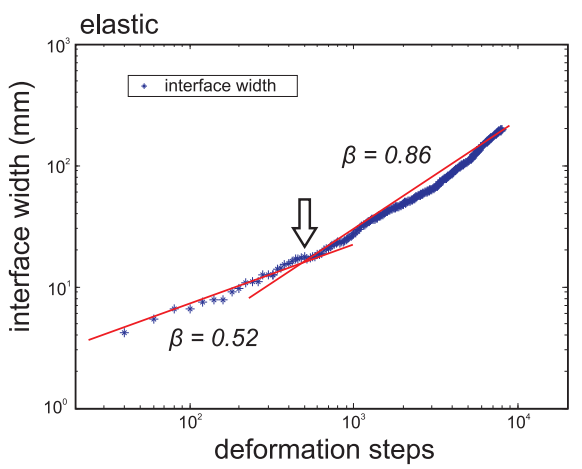

deformation steps

\section{Figure 6}

C

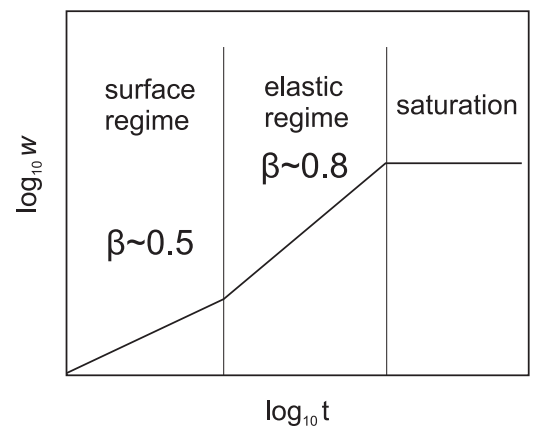

a

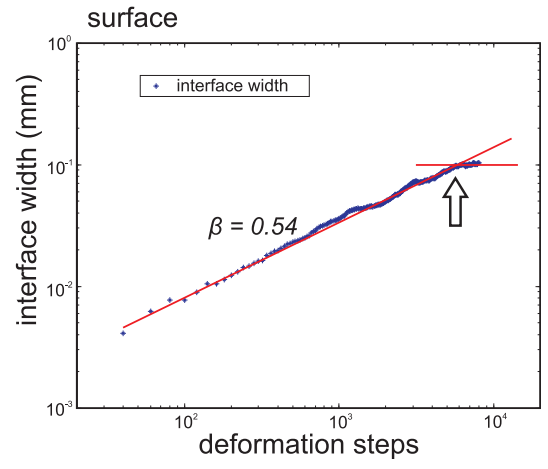


a

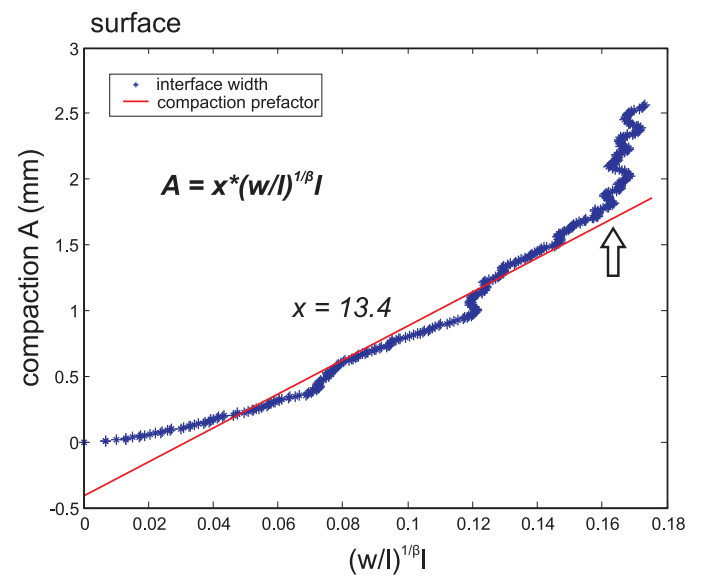

b

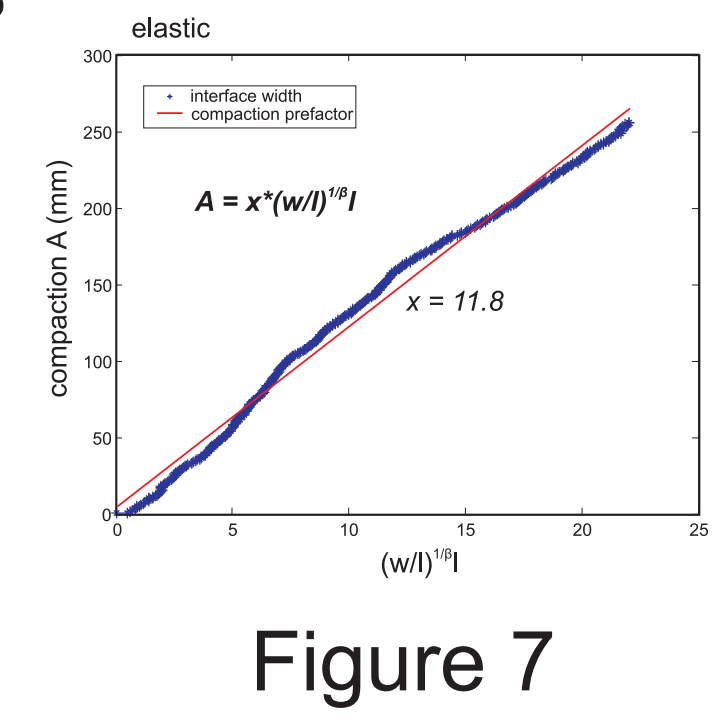

Figure 7

Figure 7 
Figure 8

a

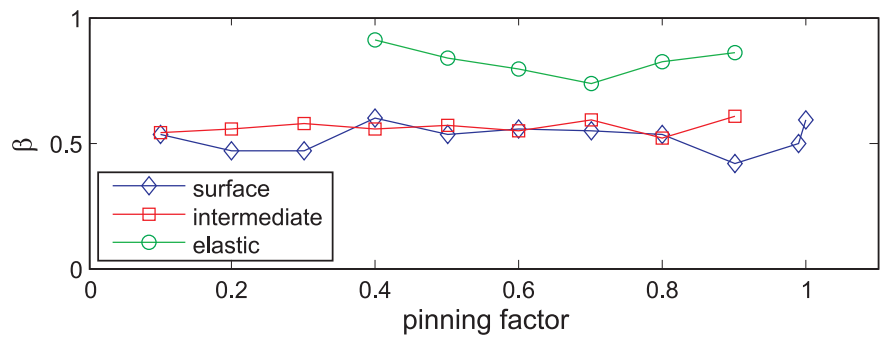

C

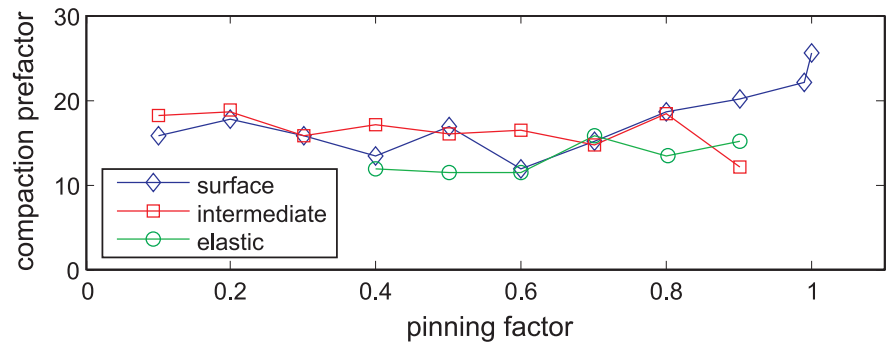

e

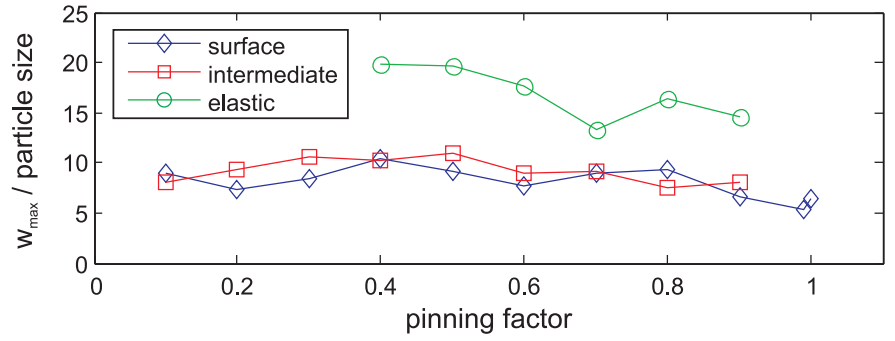

b

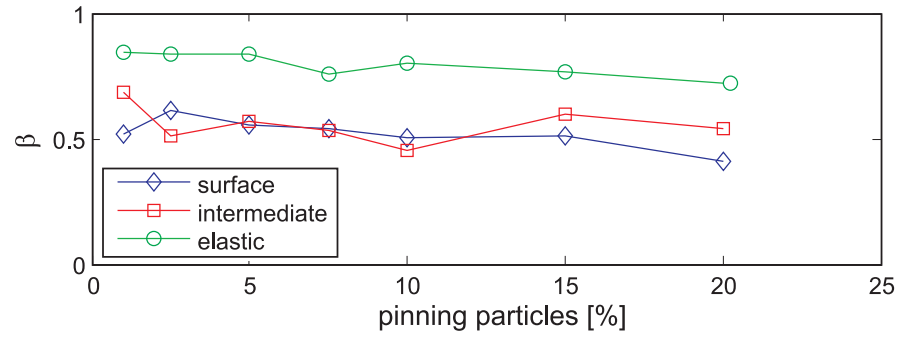

d

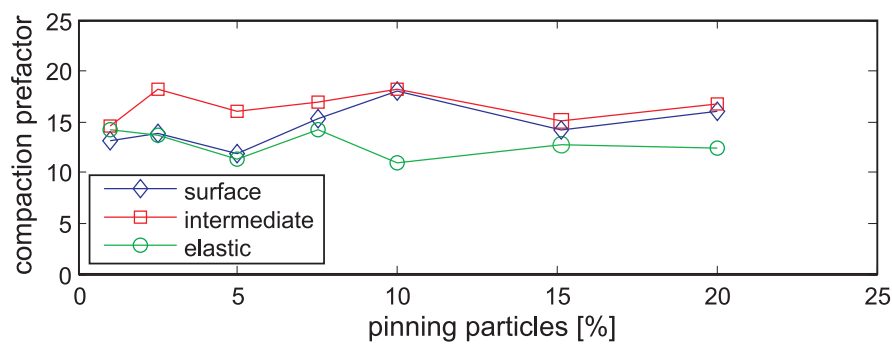

f

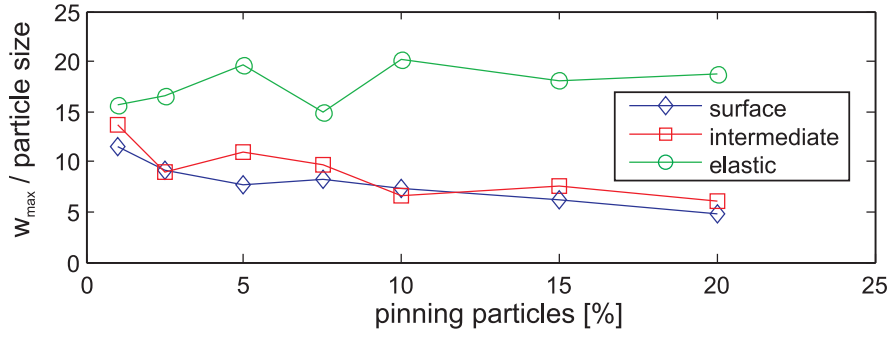

Figure 8 
Figure 9

a

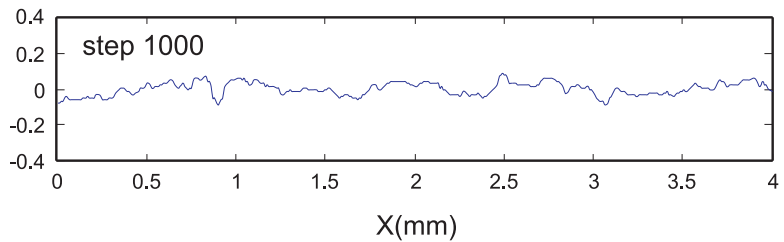

b

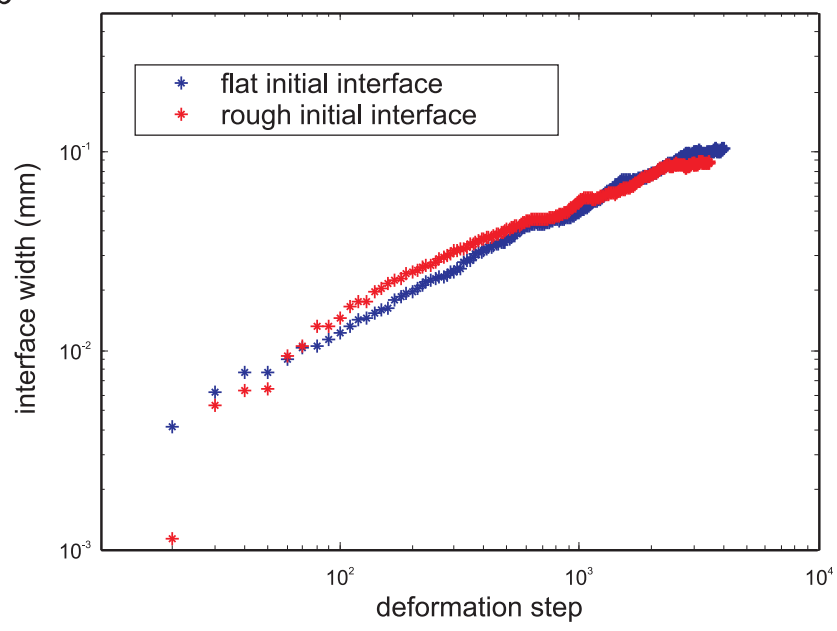

Figure 9 
Figure 10

a

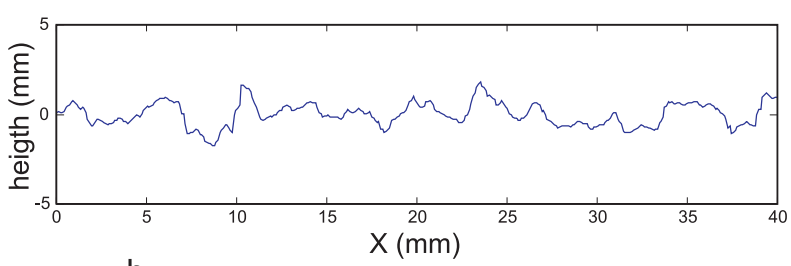

b
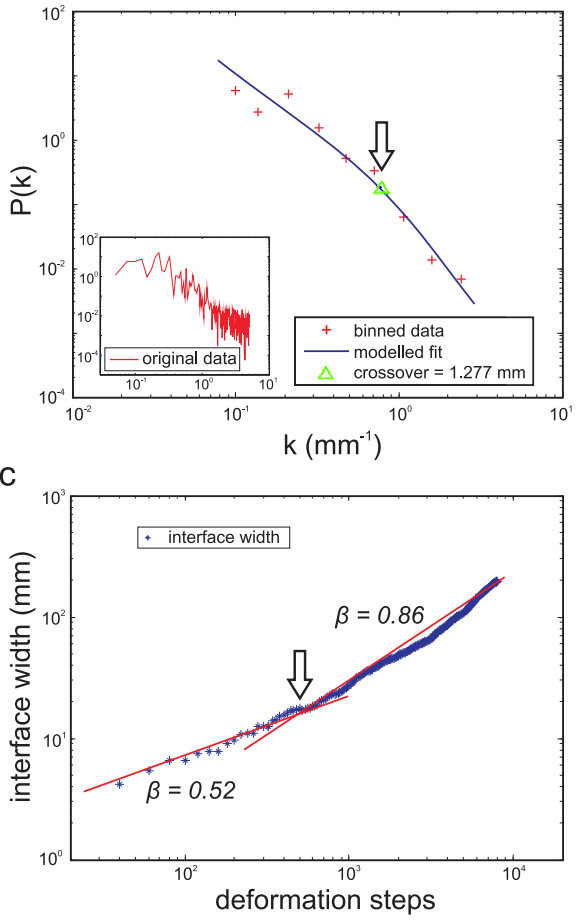

Figure 10 
Figure 11
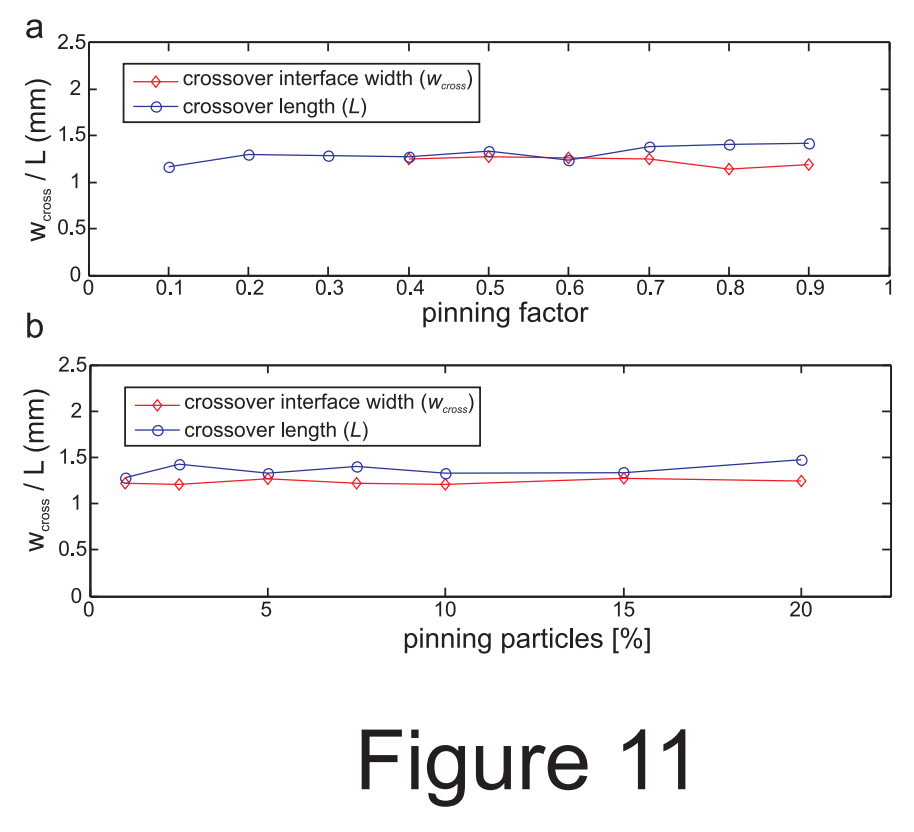

Figure 11 